Esta obra está bajo una Licencia Creative Commons Atribución-NoComercial-Compartirlgual 4.0 Internacional

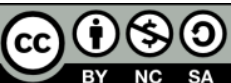

Caer en la universidad pública. Trayectorias educativas en el marco de la política pública del Albergue Transitorio Gonzalo Ricardo Mamani

DOI: https://doi.org/10.24215/16696581e222

\title{
Caer en la universidad pública. Trayectorias educativas en el marco de la política pública del Albergue Universitario
}

\section{Fall in the public university. Educational pathways within the framework of the public policy of the University Shelter}

\author{
Gonzalo Ricardo Mamani gonza.mamanisoraire@gmail.com \\ https://orcid.org/0000-0002-1457-7260 \\ Facultad de Periodismo y Comunicación Social; Universidad Nacional de La Plata (Argentina)
}

Resumen

El presente artículo indagará en torno a cómo se configuraron las trayectorias educativas de los/as jóvenes graduados/as del Albergue Universitario de la Universidad Nacional de La Plata en su paso por la educación superior y esta beca otorgada por la prosecretaria de Bienestar Estudiantil de dicha casa de estudios. La hipótesis de este trabajo intenta complejizar acerca Question, Vol. 1, N. ${ }^{\circ}$ 64, octubre-diciembre 2019. ISSN 1669-6581

Instituto de Investigaciones en Comunicación | Facultad de Periodismo y Comunicación Social | Universidad Nacional de La Plata 
Gonzalo Ricardo Mamani Caer en la universidad pública. Trayectorias educativas en el marco de la política pública del Albergue Transitorio

de los distintos marcos de políticas públicas de inclusión que lleva adelante dicha casa de estudios desde 2011 a partir de la creación de esta residencia estudiantil.

Palabras clave: universidad; políticas públicas; jóvenes; albergue universitario.

Abstract

This article will explore how the educational trajectories of the young graduates of the University Hospital of the National University of La Plata were set up during their time in higher education and this scholarship granted by the female student welfare secretary. House of Studies. The hypothesis of this work tries to make complex about the different frameworks of public inclusion policies that this house of studies has been carrying out since 2011, starting from the creation of this student residence.

Keywords: university: public politics; young people; university hostel.

El presente artículo indagará en torno a cómo se configuraron las trayectorias educativas de los/as jóvenes graduados/as del Albergue Universitario de la Universidad Nacional de La Plata en su paso por la educación superior y esta beca otorgada por la prosecretaria de Bienestar Estudiantil de dicha casa de estudios. La hipótesis de este trabajo intenta complejizar acerca de los distintos marcos de políticas públicas de inclusión que lleva adelante dicha casa de estudios desde 2011 a partir de la creación de esta residencia estudiantil.

En cuanto al referente empírico, se trata de ocho jóvenes que vivieron y se graduaron en el Albergue Universitario de la UNLP en el periodo 2016-2018. Estos/as sujetos/as son provenientes de distintas localidades y regiones del país cuya situación socioeconómica no les permita vivir en la ciudad de La Plata. En términos del abordaje metodológico, se realizó desde una estrategia cualitativa a partir de entrevistas en profundidad, la cual permitieron analizar y sistematizar a partir del método comparativo constante a los fines de dar cuenta de los procesos de las historias de vida de los/as sujetos/as.

Desde un aporte académico, este artículo forma parte del propio Trabajo Integrador Final (TIF) titulado "Jóvenes Graduados/as del Albergue Universitario de la UNLP: trayectorias, políticas públicas e inclusión" a los fines de obtener el título de licenciado en Comunicación Social con orientación en Planificación Comunicacional de la Facultad de Periodismo y Comunicación 
Social de la UNLP. Además, debe mencionarse que este TIF está enmarcado en una beca de inicio a la investigación otorgada en la convocatoria 2018 por el Consejo Interuniversitario Nacional con lugar de trabajo en el Instituto de Estudios Comunicacionales en Medios, Cultura y Poder "Aníbal Ford" de la Facultad de Periodismo y Comunicación Social, destinadas a estudiantes avanzados con más el $50 \%$ del plan de estudio de la carrera aprobada.

En las próximas líneas se desarrollarán, en un primer momento, las principales perspectivas teóricas del campo de la comunicación, la educación, políticas públicas y trayectorias educativas, seguido de las estrategias metodológicas con el cual se desarrolló este artículo. En un segundo momento, se abordará los sentidos y significaciones de cómo los/as graduados/as entienden que esta beca otorgó un marco de posibilidades para que estos/as jóvenes puedan culminar sus estudios de grado y la experiencias de las diferentes trayectorias educativas. Por último, a modo de conclusiones se dará lugar a algunas consideraciones finales de este artículo.

\section{Principales discusiones teóricas}

Este artículo recupera y realiza un aporte a los estudios de juventud que realiza la Facultad de Periodismo y Comunicación Social de la UNLP. En esa misma línea, desde el Instituto de Estudios Comunicacionales en Medios, Cultura y Poder "Aníbal Ford", posee una gran cantidad de antecedentes previos relacionados a los/as jóvenes vinculado a la educación.

Como punto de partida, este trabajo se posiciona desde el campo de la comunicación/cultura (Schmucler, 1984) ya que permite comprender las significaciones de los/as sujeto en un marco más amplio de procesos histórico social y político (Díaz Larrañaga, 2015). Schmucler expresó que es un salto teórico, que presupone el desplazamiento de las fronteras, pero que en realidad, de eso se trata, de establecer nuevos límites, definir nuevos espacios de contacto, nueva síntesis, una nueva complejidad que enriquezca y no una especialización reductora (1989).

La comunicación no es todo, pero debe ser hablada en todas partes, debe dejar de ser un objeto construido para pasar a ser un objeto a lograr. Desde la cultura ese mundo de símbolos que los seres humanos elaboraron con sus actos materiales y espirituales, la comunicación tendrá sentido transferible a la vida cotidiana" (Saintout, 2003: 80).

Si tenemos en cuenta las trayectorias educativas de los/as graduados/as del Albergue Universitario, desde esta perspectiva de la comunicación/educación podremos analizar a la 
educación por fuera a lo educativo por fuera del ámbito de la universidad y lo comunicativo a través del relato de sus prácticas, anécdotas, sus historias de vida. En ese sentido, este artículo se posiciona desde la mirada de Jorge Huergo (1994) que propuso sustituir la "y" por una barra, al igual que Schmucler con la comunicación/cultura, en la cual para significar la imposibilitar su tratamiento por separado. En ese sentido Cremona lo define "como un campo estratégico que trabaja en la relación entre la pugna por la significación y la importancia política de construir nuevas prácticas (y sentidos) colectivos frente a aquellos que históricamente se han presentado como unívocos, constitutivos y fundantes" (AA/VV, 2017: 13). Esta nació en años 60 a la luz de procesos revolucionarios en América Latina y había tensión del capitalismo triunfante posterior a la segunda guerra mundial y los movimientos políticos culturales que buscaban recuperar tradiciones culturales avasalladas en la época de la colonización y los Estados coloniales. Al mismo tiempo desde movimientos católicos producen desde la educación popular la Teología de la Liberación quien tuvo como máximo exponente a Paulo Freire (AA/VV, 2017).

Por otro lado, indagar en torno a la experiencia en la educación implica definirla. Para ello, utilizamos lo desarrollado por Buenfil Burgos (1993) quien considera que la educación no solo tiene que ver con la escolaridad. Entiende que las prácticas educativas están presentes en la constitución de los/as sujetos/as y las subjetividades de sus contextos.

Lo educativo consiste en que, a partir de una práctica de interpretación, un agente se constituye en sujeto de la educación activo incorporando de dicha interpretación algún nuevo contenido valorativo, descriptivo, conductual, conceptual, etcétera, que modifique su práctica cotidiana en términos de una transformación (Di Caudo, 2007 p.100).

Se trata de desplazarnos de los ámbitos escolares, para atender aquellos otros espacios, institucionales o no, que contribuyen a la conformación del sujeto social (Martínez, 2017: 14). En el caso del Albergue Universitario, por más que sea una institución de la universidad, pero implica la convivencia de estudiantes de todas las carreras donde se realizan prácticas más allá de concurrir a la facultad, en el cual los sujetos constituyen, reproducen o transforman las relaciones sociales vigentes. Además debe mencionarse que la heterogeneidad es mucho más amplia ya que se juntan las particularidades locales de cada uno/a de sus habitantes.

Juventud(es) 
Gonzalo Ricardo Mamani Caer en la universidad pública. Trayectorias educativas en el marco de la política pública del Albergue Transitorio

La tradición de los estudios de la juventud en la comunicación fue posterior a la institucionalización del campo en los años 80. En ese marco, desde nuestra Facultad son riquísimos la gran de cantidad de bibliografía desarrollada y también a partir de la decisión política institucional de profundizar el campo a partir de la creación del Observatorio de Jóvenes, Comunicación y Medios.

Rossana Reguillo explica que los/as jóvenes deben ser conceptualizados/as en términos socioculturales, lo que implica no conformarse con delimitaciones biológicas, y saber que no constituyen una categoría homogénea, sino que sus esquemas de representación construyen campos de acción desiguales (Reguillo, 2013).

Parto de la base de que "ser joven" es fundamentalmente una clasificación social (...) y que, al igual que toda clasificación, esta categoría supone el establecimiento de una sistema complejo de diferencia el establecimiento de un sistema complejo de diferencias, complejas, fronteras y prescripciones (Reguillo, 2013: 131).

En tanto, Margulis expresa que

Infancia, juventud o vejez son categorías imprecisas, con límites borrosos, lo que remite, en parte, al debilitamiento de viejos rituales de pasaje relacionados con lugares prescritos en las instituciones tradicionales y, sobre todo, a la fuerte y progresiva heterogeneidad en el plano económico, social y cultural (Margulis, 2008: 13).

Es necesario desencializar el concepto de "juventud", ya que esta perspectiva contribuye a introducir el principio de complejidad y a contribuir la fuerte tendencia al etnocentrismo metropolitano, al pensamiento eurocéntrico cuyas categorías son universales. Tal es el caso de moratoria social que asume que todos los jóvenes ingresan a un espacio de privilegios y excepciones que los autorizan a vivir al margen de responsabilidades, compromisos y angustias del mundo adulto (Reguillo, 2013).

También, tenemos que expresar que no hay juventud sino juventudes. En ese sentido Margulis expresa que

ser joven, por lo tanto, no depende sólo de la edad como característica biológica, como condición del cuerpo. Tampoco depende solamente del sector social a que se pertenece, con la consiguiente posibilidad de acceder de manera diferencial a una moratoria, a una condición de privilegio. Hay que considerar también el hecho generacional: la circunstancia cultural que emana de ser socializado con códigos diferentes, de incorporar nuevos modos

Question, Vol. 1, N. ${ }^{\circ}$ 64, octubre-diciembre 2019. ISSN 1669-6581

Instituto de Investigaciones en Comunicación | Facultad de Periodismo y Comunicación Social | Universidad Nacional de La Plata La Plata | Buenos Aires | Argentina

Página 5 de 27 
Gonzalo Ricardo Mamani Caer en la universidad pública. Trayectorias educativas en el marco de la política pública del Albergue Transitorio

de percibir y de apreciar, de ser competente en nuevos hábitos y destrezas, elementos que distancian a los recién llegados del mundo de las generaciones más antiguas (Margulis, 2008: 19).

Propone recuperar saber de la moratoria vital, la juventud como plus de energía o crédito temporal. A partir de allí comienza la diferencia de clase y de posición en el espacio social, lo que determina el modo en que se la procesará posteriormente. Aunque no se puede obviar ninguna de las dos rupturas objetivantes -la cronológica y la sociocultural- si se quieren evitar los peligros del etnocentrismo de clase y del fetichismo de la fecha de nacimiento.

\section{El caso de los/as graduados/as del Albergue Universitario}

En este artículo desarrolla las trayectorias de estudiantes universitarios que transitaron y experimentaron la educación desde distintos lugares, desde la gestión pública o privada, escuelas rurales y urbanas, anexas universitarias o distintos formatos de proyectos curriculares. Cabe recordar que desde la década del noventa, en la Argentina, la educación básica y media dependía de las provincias a partir de la Ley Federal de Educación 24049 (Paiva, 2016), por lo cual, la heterogeneidad es muy grande teniendo en cuenta que los ocho entrevistados son de distintos puntos del país. No obstante, no solo se verán las trayectorias en términos educativos, sino las vivencias en otros aspectos. Nos interesa conocer su la subjetividad, entendida como la articulación entre lenguaje y experiencia (McLaren, 1995) construye un soporte de representaciones que permiten significar la vivencia (Martínez, 2011). Hablar de trayectorias implica recuperar los aportes del sociólogo Pierre Bourdieu quien definió como "serie de las posiciones sucesivamente ocupadas por un mismo agente (o mismo grupo) en un espacio en sí mismo en movimiento y sometido a incesantes transformaciones" (Bourdieu, 1997b, p. 82)

En cuanto a la categoría de trayectorias estudiantiles y la diferenciación propuesta por Flavia Terigi (2007) entre trayectorias reales y trayectorias teóricas. Ello supone distinguir entre las trayectorias 0 recorridos establecidos según las leyes, condiciones normativas 0 reglamentarias, planes de estudios $\mathrm{y}$, por otro lado, los recorridos que efectivamente acontece como parte de la formación de un sujeto. Ubicar esta distinción, es la base de las condiciones institucionales, curriculares, históricas en que los estudiantes son reconocidos por las instituciones (legalmente) y por el sistema universitario como tales y no en las condiciones que los sujetos portan o carecen para poder convertirse y sostenerse como estudiantes universitarios. Esto permite necesariamente de revisar, des-naturalizar y contextualizar las 
Gonzalo Ricardo Mamani Caer en la universidad pública. Trayectorias educativas en el marco de la política pública del Albergue Transitorio

dinámicas en que las instituciones definen las trayectorias teóricas y esperables de los estudiantes a partir de las condiciones que posibilitan o restringen su acceso, regularidad, continuidad, permanencia (Ros et al., 2017).

En otro orden, se recupera los aportes de Bourdieu que conceptualizó a las trayectorias en la teoría del habitus, entendiendo que entran en juego la individualidad del sujeto y el campo de fuerzas e interacciones por el cual está atravesado en su recorrido biográfico. En ese sentido, el sociólogo francés expresa que las trayectorias son

\section{(...) una capacidad infinita de engendrar en total libertad (controlada) productos - pensamientos, percepciones, expresiones, acciones- que tienen siempre como límites las condiciones de su producción, histórica y socialmente situadas; la libertad condicionada y condicional que asegura está tan alejada de una creación de imprevisible novedad como de una simple reproducción mecánica de los condicionamientos iniciales (1991: 96).}

\section{Trabajo de campo: métodos y técnicas}

Se expresó al comienzo de este artículo que se realizaron entrevistas en profundidad a graduados/as del Albergue Universitario. Estas se realizaron en el período de diciembre 2017 y febrero 2018 a ochos jóvenes provenientes de distintas localidades del país que se pudieron culminar sus estudios a partir de la beca mencionada. Las entrevistas estuvieron sujetas no en términos estrictos a un protocolo de entrevistas a partir de cinco ejes que hacen un recorrido cronológico por sus trayectorias dentro y fuera de las instituciones educativas. En este marco, a partir de la mencionada técnica se pudo dar cuenta de toda la trayectoria educativa, desde la escuela primaria, pasando por la secundaria, el procesos de elección y cuestiones antes de conocer y transitar la educación superior y, por último, por la experiencia de vivir en el Albergue Universitario y ser los/as primeros/as graduados/as.

Para llevar a cabo el análisis de esta investigación fue necesario recurrir a una perspectiva de metodológica cualitativa ya que se busca entender que

el hombre es un animal inserto en tramas de significación que él mismo ha tejido, [...] la cultura es esa urdimbre y que el análisis de la cultura ha de ser por lo tanto, no una ciencia experimental en busca de leyes, sino una ciencia interpretativa en busca de significaciones (Martínez, 2011: 50).

Es a partir del del método comparativo constante. Este tiene como objetivo en la construcción teórica a partir de los datos obtenidos en el campo material. La selección de los materiales para 
Gonzalo Ricardo Mamani Caer en la universidad pública. Trayectorias educativas en el marco de la política pública del Albergue Transitorio

investigar deberá estar de acuerdos con los fines teóricos preestablecidos. Se interpretan los datos que se conceptualizan en el proceso de construcción de conocimientos, al vincular los emergentes reconocidos en el campo con la teoría y provee el marco para una acción potencial en el campo de lo social (Martínez, 2011).

Vasallo de Lopes explica tres principios que están presentan en la elaboración del discurso científico:

el principio de contradicción interna, que exige el modelo debe dar cuenta del tiempo lógico en el que se inscribe el discurso; el principio de la no-contradicción externa, que exige que se dé cuenta del tiempo histórico presente en la obra; el principio de la responsabilidad científica, que reclama del autor del discurso el desempeño consciente en su producción (Martínez, 2011: 51).

Además el enfoque biográfico es fundamental analizar las trayectorias educativas es necesario comprender de qué manera se fueron formando las historias de vida de estos/as jóvenes. Además, permite poner en valor la diversidad de sentidos atribuidos por los actores sociales a los acontecimientos vitales por los que han atravesado, en un contexto social en el que prima la diversidad de adscripciones y las referencias, muchas veces, contradictorias entre sí (Kornblit, 2007).

Como explica María Rosa Cifuentes Gil, en el marco de la intervención social, estas historias ayudan a entender la vida de personas y de colectivos, las condiciones en las que crecieron y se desarrollaron, el modo en el que toman las decisiones y los factores históricos y psicosociales que influyen en su entorno (Cifuentes Gil, 2011).

En cuanto a las técnicas de investigación que se utilizaron para el relevamiento etnográfico fueron: la entrevista en profundidad, entrevistas semiestructuradas y registro escrito.

Marradi, Archenti y Piovani explican que la entrevista en profundidad es

un proceso comunicativo por el cual el investigador extrae una información de una persona. Esa información es el conjunto de representaciones asociadas a acontecimientos vividos por [él]. Destacamos el rol mayéutico y estratégico del entrevistador. Su principal función es la de escuchar, y recordamos que la información que provee el entrevistado no debe apreciarse en términos de veracidad-falsedad sino como el producto de un individuo en sociedad cuyos relatos deben ser contextualizados y contrastados (2007: 218-219).

En cuanto a la entrevista semiestructurada, Marradi propone que esta debe a partir de un estímulo particular, en la que se narren historias de la vida cotidiana y que el entrevistador 
Gonzalo Ricardo Mamani Caer en la universidad pública. Trayectorias educativas en el marco de la política pública del Albergue Transitorio

cuente al entrevistado para luego explorar sus reacciones y reconstruir sus preferencias valorativas (Marradi, Archenti y Piovani, 2007: 218).

\section{Protocolo de entrevista}

Estuvo conformado a partir de cinco ejes: familia; educación primaria; educación secundaria; universidad; vínculo con el Albergue Universitario. Se buscó obtener la mayor cantidad de información posible respecto a su trayectoria biográfica poniendo foco no tan solo en la cuestión educativa sino todo lo que conlleva ir a la escuela y a su vez otras instituciones que consideren que fue formativa en sus vida, por ejemplo clubes, centros de fomento, iglesia, entre otras. En ese sentido se buscó que los/as entrevistados/as utilicen el relato de la anécdota para recrear las escenas.

A continuación se detallan sus nombres -cambiados por una cuestión confidencial- sus lugares de origen y su tiempo en el lugar. Debe aclararse que solamente los nombres fueron cambiados y acordado con los/as entrevistados/as

-Carolina: 2014-2016. Trelew, Chubut. Periodista Deportivo.

-Cande: 2011-2017. Purmamarca, Jujuy. Odontóloga.

-Darío Paz: 2012-2015. Posadas, Misiones. Ingeniero civil.

-Tomás Ruiz: 2011-2015. Tandil, Prov. Buenos Aires. Diseño en Comunicación Visual.

-Pía Mansilla: 2012-2014. Pergamino, provincia de Buenos Aires. Profesora en Comunicación Social.

-Marcos: 2012-2016. Magdalena, provincia de Buenos Aires. Administración de Empresas.

-Matías: 2012-2017. Santo Tomé, Santa Fe. Licenciado en Astronomía.

-Fabiana: 2014-2017. Mar del Plata, provincia de Buenos Aires. Licenciada en Artes Plásticas.

En el primer eje el protocolo buscó centralizar en la composición familiar, los quehaceres de cada integrante, ya sea laboral o educacional. Además buscar que los/as entrevistados/as cuenten qué recuerdos tienen de la convivencia que dejaron de compartir puesto que tuvieron que emigrar de su ciudad/pueblo. En cuanto a este último punto, si su proceso de independización fue dificultoso. Por último, teniendo en cuenta lo histórico social y político de nuestro país si tienen recuerdo de la crisis del 2001. 
Gonzalo Ricardo Mamani Caer en la universidad pública. Trayectorias educativas en el marco de la política pública del Albergue Transitorio

\section{Experiencias en el Albergue Universitario}

Vivir, convivir y experimentar el Albergue Universitario fue un recorrido que demandó varios años estudiantes. Son la primera generación de graduados/as que dio la política pública desde su inauguración en 2011. El primer caso fue en 2014 de un estudiante avanzado de Mar del Plata y luego vinieron estos/as jóvenes. Es el caso de Candela, Tomás y Matías que hicieron toda su carrera dentro del Albergue y se recibieron en el tiempo estipulado por los planes de estudios de sus carreras. El resto entraron con un grado avance en la carrera y vivieron en distintos lugares, con la excepción de Marcos que viajaba desde Magdalena (a 60 kilómetros al sur de La Plata) para cursar.

En primer lugar, los/as entrevistados/as, conocieron a la beca del AU a partir de internet, por medio de conocidos/as que habían escuchado la noticia o tenían conocimiento y folletería de la secretaría de extensión de las facultades. En aquel momento, la prosecretaria de Bienestar Universitario de la UNLP estaba denominada Asuntos Estudiantiles y era la encargada de la difusión del AU.

Dentro del primer año de AU, la inscripción a la beca fue para 36 estudiantes en la cual estuvieron Candela, Matías, Darío y Marcos. Ellos/as comentan que inaugurar el albergue fue el mejor año que vivieron dentro de su transcurso. Al ser pocos estudiantes, había una unión del grupo consolidado, pero que se perdió en el segundo ante la primera crisis institucional que vivió el $\mathrm{AU}$, que tuvo como consecuencia la expulsión de un estudiante becado y la renuncia de otra. A partir de este hecho se produjo un quiebre entre los/as becarios/as y las autoridades del mismo. En ese sentido se nota puede notarse el posicionamiento de los/as becarios/as en su discurso.

En este segundo año entraron nuevos/as becarios/as, entre ellas Pía junto a su hermana, ambas quedaron seleccionadas en la misma convocatoria. Esto es interesante recalcar porque la beca no contempla cupos por familia sino la condición económica de la misma.

En 2014 se inauguró el segundo edificio habitacional (módulo uno) que produjo una gran cantidad de ingresantes a la beca. En esta convocatoria obtuvieron la beca Carolina y Fabiana. A partir de este año, comienza un proceso de masificación e institucionalización del AU, ya que no solo entraron nuevos/as estudiantes, sino que se amplió la cantidad de trabajadores/as y recursos. Este proceso de masificación es visto desde la primera camada como un proceso positivo ante la cantidad de nuevos/as ingresantes, pero negativo ya que hay falta de conciencia de la comunidad de lo que significa el AU.

La masificación e institucionalización del AU con el correr del tiempo, se dieron procesos de mayor alcance de la beca, a partir de la organización de los/as estudiantes. Podría decirse que

Question, Vol. 1, N. ${ }^{\circ}$ 64, octubre-diciembre 2019. ISSN 1669-6581

Instituto de Investigaciones en Comunicación | Facultad de Periodismo y Comunicación Social | Universidad Nacional de La Plata La Plata | Buenos Aires | Argentina

Página 10 de 27 
Gonzalo Ricardo Mamani Caer en la universidad pública. Trayectorias educativas en el marco de la política pública del Albergue Transitorio

el hecho fundacional de estos procesos de cambio convivencial a medida que se iban formando acuerdos con las autoridades y entre los/as becarios/as. En ese sentido se amplió los derechos de la beca, desde carga de SUBE (cuando no estaba la ley provincial del boleto educativo), pasajes de larga distancia en el receso vacacional, nuevas computadoras, mejoras de la conexión de internet, el no cierre del AU en vacaciones de invierno, mayor cantidad de bicicletas, proyector y biblioteca etc. Fundamentalmente debe remarcar la cuestión de la convivencia puesto que según los/as graduados/as se manejaba un reglamento que no estaba acorde a la realidad de los/as becarios/as y fue creado por personas externas al día a día del $\mathrm{AU}$.

Los/as estudiantes: lugar central en la lucha y conquistas de derechos

El Albergue Universitario nació por la lucha constante de los/as estudiantes universitarios que buscaban un lugar en la cual puedan permanecer durante los meses de clases en la ciudad. También en una alimentación económica y nutritiva para atravesar la rutina de las cursadas. No obstante para que todos/as puedan acceder a la universidad, el boleto educativo gratuito. Todas estas demandas se las nombró durante años como "Reindivicación ABC: Albergue, Boleto y Comedor". Estos derechos fueron conquistados durante el período 2003-2015. En tiempos actuales, donde la inflación está consumiendo el salario real de los/as trabajadores, el movimiento estudiantil logró un nuevo derecho: el comedor turno noche y las actualizaciones de las carga de tarjeta SUBE ante los aumentos en el precio del boleto.

En este apartado se analizará las valoraciones de los/as graduados/as del rol del movimiento estudiantil como actor fundamental para la conquista de derechos, comprendiendo que pudieron graduarse a partir de una conquista estudiantil.

En términos generales, los/as entrevistados/as reconocen la labor de los centros de estudiantes y la lucha de los/as estudiantes como actores clave. Lo manifiestan como una gran ayuda y sostén para llevar bancar la carrera. Además que por más que sean gratuitas y sin ingresos, aun así es casi imposible estudiar, por lo cual este derechos fue clave, más allá que la beca sea mínima como las que otorgan los municipios, es valorada. De estos/as ocho entrevistados/as solo es el caso de Matías que militó en una agrupación estudiantil en la Facultad de Astronomía:

Sin embargo por otro lado, la politización del mismo es muchas veces es cuestionadas:

Creo que el albergue te da eso pero también uno termina comprendiendo y lo que significa la conciencia social que uno tiene que con la universidad pública y el albergue. No es un 
Gonzalo Ricardo Mamani Caer en la universidad pública. Trayectorias educativas en el marco de la política pública del Albergue Transitorio

regalo, sino una conquista de estudiantes que estuvieron antes que nosotros. Como el boleto, no es un regalo, es un derecho, La educación es un derecho y el albergue va en pos de ese derecho (Tomás Ruiz, comunicación personal).

\section{Pensar, dialogar y redescubrir el Albergue}

Desde 2016 hasta la actualidad en el AU conviven 130 estudiantes provenientes de distintos lugares del país y otros países latinoamericanos, la diversidad cultural es enorme y convergen en un solo lugar. Este punto de encuentro de estudiantes provocó procesos de comunicación/educación desde distintas dimensiones y llegadas. Durante su segundo año ante la crisis convivencial se produjeron distintas asambleas para poner un límite a la imposición del reglamento. Luego, en 2014, con la llegada de una gran cantidad de ingresantes, se realizaron distintas performances artísticas y jornadas culturales por parte de albergados/as de Bellas Artes y otras facultades. En 2015 se ampliaron los derechos que hacen a la beca del albergue, mientras que en 2016 se continuaron con las actividades artísticas con la creación de murales, peñas folclóricas y jornadas al aire libre por el día del estudiante. Por último, el año pasado con la apertura del AU para llevar a cabo prácticas preprofesionles con estudiantes de distintas facultades, entre ellas la Facultad de Periodismo y Psicología. Esto último es fundamental, porque el $\mathrm{AU}$ debe relacionarse con lo que sucede en el mundo universitario y un no un lugar aislado en la cual se vive, pero debe reconocerse que estos son primeros pasos.

Esta síntesis fue lograda por la articulación de los/as estudiantes del AU y las autoridades del mismo formando un trabajo en conjunto y abierto. Las continuidades de este proceso que comenzó en 2012 hay que analizarlo entendiendo principalmente el contexto, la demanda de los/as estudiantes y la ampliación de derechos a partir de la participación democrática de la comunidad.

Con respecto al paso de estos/as graduados/as expresan que el vínculo entre los/as compañeros/as, en un primer momento estos tuvieron la tendencia a la sociabilización con otros, conocer, aprender, vincularse, salir y el estar unidos/as. No obstante con el paso de los años estos tienden a la lejanía, donde dieron lugar a los vínculos por "afuera del albergue" como si fuera un lugar cerrado y sin apertura al exterior. En el ese marco, la primera camada es fuerte esta lejanía ya que, reciben con beneplácito el ingreso de nuevos/as becarios/as, pero ante la masividad sienten nostalgia del primer año. 
Gonzalo Ricardo Mamani Caer en la universidad pública. Trayectorias educativas en el marco de la política pública del Albergue Transitorio

El día a día: las nociones con respecto al espacio

En los primeros momentos y hasta 2014 en el AU, las habitaciones distribuidas en los módulos habitacionales fueron compartidas por estudiantes en ocho de las once habitaciones eran compartidas por dos becarios o becarias (mismo género). Los baños están ubicados en la parte central del pasillo estaban repartidos en cuatro: duchas, lavamanos e inodoros.

Sin embargo desde de la inauguración módulo uno, tras la decisión del Presidente de la UNLP, Fernando Tauber (2010-2014), las habitaciones pasaron a ser individuales, lo cual generó una mayor comodidad y privacidad de los/as becarios/as, aunque una disminución significativa de la cantidad de plazas disponibles, puesto que el proyecto original y cuando el AU estaba en manos de privados, era de 400 personas; actualmente se prevé un máximo de 200 plazas. Esta decisión para los/as graduados/as fue importante ya que pudieron acceder a un espacio propio a partir de una habitación individual, en la cual pudieron disponer de su organización y distribución del tiempo para el descanso, estudio, ocio, etcétera. Además de evitarse la problemática de convivir con otra persona en un mismo cuarto. Por otra parte, puede notarse la valoración por la magnitud del espacio verde en los patios entre los módulos y trasero en la cual se utilizan para la distracción y el deporte ya que cuenta con canchas de fútbol, vóley y básquet. Además de contar parrilla y mesas, alto sumamente demandado y tras una fuerte discusión se logró.

La gran mayoría de estos/as jóvenes ya habían emigrado de sus casas en temprana edad por lo cual no sufrieron un fuerte desarraigo de sus lugares de origen. Esta poca dependencia fue solamente económica pero con el correr del tiempo fue menor. Los/as graduados/as buscaron desde la finalización del secundario la independencia de los padres.

Igualdad de oportunidades no es sinónimo de mérito

Hablar de políticas públicas aparece transversalmente la noción de la meritocracia y el esfuerzo en el momento del acceso o en la evaluación de renovación de las becas en general y en particular del AU. Estos discursos van en la misma línea en la cual el actual gobierno nacional entiende a las carreras estratégicas y ofrece un aumento mayor de dinero en la reconfiguración y ajuste de la becas de inclusión educativa, tal es caso de la PROGRESAR. A continuación de algunos fragmentos de las entrevistas:

También pasó que en el lapso que le renovaban a cualquiera y no aplicaron el criterio del mérito (Marcos, de Magdalena, comunicación personal).

Question, Vol. 1, N. ${ }^{\circ}$ 64, octubre-diciembre 2019. ISSN 1669-6581

Instituto de Investigaciones en Comunicación | Facultad de Periodismo y Comunicación Social | Universidad Nacional de La Plata La Plata | Buenos Aires | Argentina

Página 13 de 27 
La verdad que creo que es una buena oportunidad, pero es una política que tienen que regular bien. Acá en el albergue está fallando en el sentido de a quién. Nosotros no sabemos a quién se le otorga, porque se le otorga, los chicos que están adentro controlarlos. Saber que cuando se le renueva, si se lo siguen mereciendo. Si realmente eso. Que sea una política bien regulada (Candela, de Purmamarca, comunicación personal).

Cuando entré había cinco o seis chicos que estudiaban ingeniería dentro de los 100 pero para mí eran muy pocos. Creo que el albergue al depender de la Universidad tendría que, no es por desmerecer nada, pero debería priorizar carreras como la de ingeniería, conozco un montón de chicos que quedaron afuera por diferentes motivos, y otra gente que quedó luego abandonó o se fue. Por eso creo que habría que hacer un debate para priorizar las carreras de ingeniería, medicina, científica, carreras que aporten al país (Darío, de Posadas, comunicación personal).

Estos/as jóvenes hablan particularmente del caso del AU en cuanto a la noción del mérito y el esfuerzo, lo que da como consecuencia la crítica hacía la política pública puesto que si tenemos en cuenta los objetivos del mismo, además de asegurar el ingreso, permanencia y egreso de los/as estudiantes a partir de la igualdad de oportunidades, debe fomentar la conciencia de lo público, la participación activa de los/as alumnos/as y la justicia social. Si se entiende a esta política pública como meritocracia y a los fines de fomentar carreras estratégicas, pierde la misión que fue planteada a partir de su creación y sus objetivos. Desde la universidad y quienes administran esta beca deben generar mecanismos participativos con los/as miembros de la comunidad a los fines de construir sentido de lo que significa este derecho.

Por otra parte, si se tiene en cuenta las nociones y percepciones de lo que entienden los/as entrevistados/as por igualdad de oportunidades y los procesos de adjudicación y renovación de la beca del $A U$, ellos/as quizás no hubiesen podido finalizar su carrera universitaria por los distintos motivos que consideran importante aplicar a los/as nuevos/as ingresantes. Los criterios que los/as jóvenes consideran importantes para permanecer en este derecho los excluiría a varios/as por los motivos de meritocracia, carreras prioritarias y rigurosidad académica. 
Gonzalo Ricardo Mamani Caer en la universidad pública. Trayectorias educativas en el marco de la política pública del Albergue Transitorio

\section{Rendimiento académico y exigencias del Albergue}

Durante los primeros años del AU, el mismo establecía en su reglamento general que los/as estudiantes no podían dedicarse a trabajar porque se consideraba que el/la estudiante tenía todo cubierto para dedicarse exclusivamente a estudiar argumentando que había becas de ayuda económicas nacionales. Sin embargo, en primer lugar esto no se cumplía porque no había una método de control si el trabajo era informal, en segundo lugar, los/as estudiantes obtenían becas de trabajo de los centros de estudiantes (fotocopiadora o buffet) ya que muchas veces sus padres no podían sostenerlos económicamente y, tercer lugar fue otra victoria de los/as becarios/as puedan trabajar y estudiar viviendo en la política pública.

Esta introducción es necesaria porque la primera camada de graduados/as lo vivió y tuvieron que amoldarse a la situación que desde la Presidencia de la UNLP se exigía. Mientras que la segunda estuvo en la desreglamentación de esta.

Para la renovación de la beca que se hace anualmente, deben haber aprobado aproximadamente el $14 \%$ plan de estudios de su carrera, es decir que un estudiante de Periodismo debería aprobar o promocionar cinco de las siete materias que puede cursar por año, del plan de estudios del año 98. A su vez, haber realizado una entrevista con el equipo psicopedagógico para relevar los procesos educativos durante el año y en relación a los anteriores. Existe la salvedad en la práctica que si el/la estudiante es ingresante a la universidad pueda realizar -por única vez- el cambio de carrera.

En cuanto a la experiencia de los/as primeros/as graduados/as más allá de esta cuestión reglamentaria expresan que tuvieron un cambio radical en su procesos formativos.

En el albergue comía y descansaba, mejoré las notas, venía bien, tenía el tiempo para socializar. Me re ayudo, en algún momento me iba a cansar de viajar todos los días. Es un sacrificio pero en 4to año se pone jodido. Cuando entre al albergue me puse a leer, socializar, que nos seguimos viendo, vienen a tomar mates, y yo me fui a orientando a más ciencias sociales (Marcos, de Magdalena, comunicación personal).

Las preocupaciones por la economía, lo laboral, el alquiler y otras cuestiones con respecto a ser independiente o dependiente de los padres son escollos que los modos de ser estudiante universitario pasan por la cabeza e impide la concentración.

Es otra de las ventajas, me pude dedicar al 100\% a estudiar. Volviendo a tener gastos constantes, alquiler, servicios, comidas, mi vieja me podía mandar algo que lo usaba para fotocopias u otros gastos que tenía (Carolina, de Trelew, comunicación personal).

Question, Vol. 1, N. ${ }^{\circ}$ 64, octubre-diciembre 2019. ISSN 1669-6581

Instituto de Investigaciones en Comunicación | Facultad de Periodismo y Comunicación Social | Universidad Nacional de La Plata 
El hecho de existía el albergue, que me daba una re herramienta de no tener que laburar a la par de la carrera, me da una estabilidad económica que mi vieja no me podía garantizar (Matías, de Santo Tomé, comunicación personal).

En el caso de la mayoría estos/as jóvenes dejó de ser gran parte una cuestión importante, aunque para otros/as siguió presente ya que los materiales de su carrera son muy altos y es necesario tenerlo en cuenta porque si no les imposibilitaba cursar y estar a la altura de las exigencias. Carreras como odontología, artes plásticas y diseño en comunicación visual (dentro de los casos relevados) requieren grandes costos en las herramientas de prácticas, por lo cual el AU fue una ayuda sumamente necesaria.

Es muy cara la carrera. No sé, porque realmente si yo hubiese querido, mis viejos hubiesen puesto todo de ellos para que terminara. La ayuda del albergue es un montón, te facilita un montón de cosas, así que la verdad que no hubiese podido. (..) Al principio el albergue estableció que no podías trabajar, que tenías que dedicarte puramente a la facultad, pero en 3ro cuando empieza la compras de materiales para las clínicas, empecé a trabajar limpiando casas, hasta que mi cuenta que no podía por el tiempo y los parciales (Candela, comunicación personal).

El aporte de Darío en cuanto a las facilidades que le dio el albergue está vinculada a la cuestión alimenticia. En el AU están garantizadas las cuatro comidas durante todo el año, incluso en vacaciones de invierno, en el cual cuentan con un menú -tradicional y vegetarianoque está planificado por un nutricionista que elabora una dieta para un/a joven universitario/a. Por lo cual los/as becarios/as no tienen que preocuparse por los gastos de comida, ni perder tiempo en la elaboración de la misma. Por otro parte, al contar con un amplio espacio verde, los/as estudiantes organizan partidos de fútbol, voley y yoga como forma de esparcimiento de la rutina.

En la comida, calidad de vida, tiempo. La comida que te dan acá, no te la dan en cualquier otro lado y eso ayuda mucho, ya sea en el crecimiento, para estudiar bien, para estar sano. El lugar de esparcimiento que tiene, también ayuda mucho en la hora del estudio (Darío, de Posadas, comunicación personal). 
Gonzalo Ricardo Mamani Caer en la universidad pública. Trayectorias educativas en el marco de la política pública del Albergue Transitorio

\section{Experiencias similares de albergues}

En el transcurso de este Trabajo Integrador Final se pudo investigar exploratoriamente acerca de otras experiencias similares de becas de inclusión universitaria. Pero también se produjo, por medio de las entrevistas, que los/as graduados/as comenten acerca de estos y su mirada de los mismos. Asimismo fueron las conversaciones con Tomás y Carolina quienes introdujeron esta cuestión y marquen una posición con respecto a la falsa alusión de que el caso de la UNLP sea el único.

Carolina comentó que la Universidad Nacional de la Patagonia Juan Don Bosco donde cursó sus primeros años luego del secundario, la existencia de gamelas universitarias. Por otro lado, la Universidad Nacional del Centro de la Provincia de Buenos Aires posee un albergue universitario en el cual viven 150 estudiantes. Las otras experiencias se tratan del Albergue Estudiantil de La Rioja y la Residencia Universitaria en Santiago del Estero.

Hay un especie de mito acerca del mito que el albergue de la UNLP es el único en Argentina. No es así, la Universidad Nacional de la Patagonia cuenta con, no se llama albergue, pero son gamelas que le dicen pero también una especie de pensión, con mucha menor capacidad pero para chicos del interior de Chubut que van a estudiar como yo. Es cierto que no es totalmente gratuito, pero por lo menos en el alojamiento si y los servicios también (Carolina, de Trelew, comunicación personal).

En Tandil hicieron un albergue similar por ejemplo, es pata del derecho de poder acceder a la educación universitaria. No alcanza que la universidad sea gratuita hoy, sino que los chicos necesitan que les brinden espacio para vivir. Hay varias situaciones en el albergue universitario nuestro. Hay chicos que prácticamente estaban en situación de calle (Tomás, de Tandil, comunicación personal).

En cuanto a políticas de ayudas a estudiantes universitarios se pudo dar cuenta que dos graduadas vivieron en Casas de Estudiantes. Si bien estas cada una tienen sus lógicas de funcionamiento y no hay lógica universal, las entrevistadas comentaron que la municipalidad les otorgaba cierta ayuda. En la ciudad de La Plata son decenas de casas municipales y provinciales en la cual estudiantes viven y realizan sus estudios universitarios y terciarios. 
Gonzalo Ricardo Mamani Caer en la universidad pública. Trayectorias educativas en el marco de la política pública del Albergue Transitorio

Vine a la UNLP porque acá está la casa de Mar del Plata. Podía venir y pagar un alquiler económico y a la vez estudiar. Era un edificio de tres pisos que compartíamos cocina y baño... éramos como 40, cada uno tenía su espacio....Éramos 20, después 15,10 y duró lo que duró...era una beca estar ahí (Fabiana, de Mar del Plata, comunicación personal).

Esa casa del estudiante la solventaba ex estudiantes de Pergamino recibidos que habían estado acá en La Plata. El que la fundó había fallecido, como si fueran 5 chicos del albergue se egresan y el día de mañana decide fundar otro espacio chiquito de sus ciudades acá. Entre todos las mantenían. La municipalidad creo que muy poco, no me acuerdo, creo que para algún impuesto (Pía, de Pergamino, comunicación personal).

En este punto todos los/as graduados/as llegaron a la conclusión que es necesario que estas experiencias de políticas públicas de inclusión universitarias se repitan en otras universidades públicas, sobre todas en aquellas de alcance masivo y reciban estudiantes de distintas lugares país y otros países, como son los casos de la UNC, UNR, UNT, entre otras. Pero fundamentalmente es necesario llevar la universidad a los sectores populares donde, como es el caso de estos/as jóvenes, sea la primera generación de estudiantes graduados en sus familias.

\section{Políticas Públicas nacionales destinadas a la universidad}

Durante el período 2003-2015 las universidades públicas tuvieron un crecimiento sostenible que se tradujeron en obras de infraestructuras, aumento de carreras de grado y posgrados, nuevas universidades, mejora salarial docente, incremento en el presupuesto de ciencia y técnica, etcétera. Pero en cuanto a los/as estudiantes fueron creadas becas para el incentivo y respaldo en la educación superior. En ese marco, todos los/as graduados/as obtuvieron estos nuevos derechos, principalmente las becas PNBU y PNBB, y en menor medida la PROGRESAR puesto que empezó en 2014 cuando estos ya se encontraban en gran avance en la carrera y en algunos casos fueron transferidos. Además la limitación de la edad (hasta 24 años) fue un impedimento. También es necesario mencionar que se manifestaron experiencias con becas provinciales y municipales, pero que eran compatibles con las de bajada nacional por lo cual no podían acceder a ambas.

Los/as graduados/as expresan que las mismas les representaban como una seguridad, respaldo, una ayuda en cuanto a sus gastos personales a la hora de poder concurrir a la Facultad. Sin embargo reconocen que los pagos en PNBU no eran como estaba planteada (10 cuotas a cobrar del 1 al 10 dependiendo el DNI) pero que en la práctica eran dos al año, a 
Gonzalo Ricardo Mamani Caer en la universidad pública. Trayectorias educativas en el marco de la política pública del Albergue Transitorio

diferencia de PROGRESAR donde había una regularidad en los pagos. Este detalle es importante porque hace a la cotidianidad de los/as estudiantes en la cual los gastos promedios son importantes. Esto se refleja en carreras como Odontología en la cual el gasto de instrumentales y demás. A pesar de ello el valor que representa la adjudicación de este tipo de becas que en dos graduados/as permitió el acceso a una computadora personal, algo que al mismo tiempo se estaba llevando a cabo en las escuelas secundarias a nivel nacional por medio del Programa Conectar Igualdad. Por otra parte, permitió una autonomía económica ya que a partir de esta entrada, posibilitaba no depender de la economía de los padres o no tener que trabajar demasiadas horas de trabajo.

Recibía una beca que no se acuerdo el nombre. Estaba con el gobierno anterior la otra beca. Me pude comprar una pc para el último año, de ahorrando me compraba un calzado o ropa. Un año y medio la recibí... (Pía, de Pergamino, comunicación personal).

Con la PROGRESAR pude comprarme la pc que era necesaria. Después mis papás me ayudaban pero una risa porque tenía otro valor el precio, rendía un poco más. La beca del municipio era de 150 pesos y mis papás me mandaban 200 pesos. Con eso tiraba... (Tomás, de Tandil, comunicación personal).

Lo inédito es un caso acerca de la política pública de la UNLP de pueblos originarios, en la cual jóvenes provenientes de Salta y Jujuy pudieron venir a realizar una carrera universitaria a partir del Albergue Universitario. Sus padres nunca tuvieron la chance de estudiar la universidad. Su madre es vendedora de productos regionales en la plaza principal del pueblo y su padre un policía retirado que se dedica a la construcción. Es la única mujer entre cuatro hermanos que tampoco pudieron entrar a la universidad por varios motivos, salvo uno de ellos que se encuentra en la escuela secundaria.

Esta beca surge en 2009, principios en enero, todo de casualidad. Es un proyecto que lanzó inclusión de pueblos originarios a la universidad, las que estaban anotando o buscando pueblos originarios en el norte, cayeron de casualidad a mi pueblo y justo en hostel donde estaban era el señor que manejaba al principio mi comunidad. Entre charla surge e hizo una reunión, se informó más o menos lo que era. Era una beca que incluía estadía, transporte, ayuda económica, inscripción (Candela, comunicación personal).

Es necesario mencionar que el Programa Conectar Igualdad no fue nombrado pero sí reconocido por los/as jóvenes puesto que estos/as egresaron del colegio secundario 
Gonzalo Ricardo Mamani Caer en la universidad pública. Trayectorias educativas en el marco de la política pública del Albergue Transitorio

justamente en el periodo en el que fue implementado e incluso antes. Este programa de inclusión digital educativa tenía como objetivo disminuir la brecha entre quienes tienen acceso a una computadora personal.

\title{
Etapa final como becario: ¿Y ahora? ¿Cómo se empieza de nuevo?
}

Una vez graduado/a un estudiante becario del AU ¿cómo se organiza la salida de la beca? ¿Qué hicieron estos/as jóvenes? ¿Qué fue de sus vidas? ¿Qué hizo el AU para acompañarlo en su finalización de beca?

El destino de los/as jóvenes fue diverso. Algunos/as consiguieron trabajo rápidamente, otros/as regresaron a sus pueblos/ciudades por motivos varios y en dos casos la dirección del AU articuló con sectores de la universidad para la inserción laboral. En todos los/as entrevistados/as coincidieron que salida laboral y el qué hacer una vez graduado/a es un nuevo escollo para sus vidas. La finalización de la carrera universitaria abrió en sus vidas nuevos desafíos y oportunidades, pero indudablemente una crisis personal.

En el caso de Darío consiguió trabajo rápidamente luego de haberse graduado de Ingeniero civil y pudo insertarse en la vida independiente fácilmente, aunque no niega la dificultad de haber ido del AU. Por el lado de Marcos y Tomás ante la dificultad de no conseguir trabajo, la dirección del AU consideró tenerlos en cuenta para gestionar con otros espacios de la universidad para que puedan insertarse a partir de una beca trabajo. En el primer caso no aceptó porque había ganado un concurso en la Fuerza Aérea, mientras que Tomás accedió a la beca por lo que luego quedó efectivo en el cargo.

En este sentido Tomás reflexiona acerca de la inserción laboral de los/as graduados/as y el rol del AU como política pública de inclusión:

\begin{abstract}
Algo que al albergue le falta gestionar, que es el futuro graduado se inserte en el ámbito laboral. Porque si no te ves en un lugar sin salida que te queres matar, yo estaba en 4to, no había perspectiva de cómo conseguir trabajo y no conseguía. Hasta que trabaje en un boliche en un guardarropa y ahí empecé a tener plata para mí y pude ahorrar. Es algo importante, ahorrar para tener un lugar. Después conseguí un laburo en la universidad. En secretaría de arte y cultura, que fue un golazo pero también hubo como intermediario la gestión de Bárbara. Tenemos a estos futuros graduados, para que se puedan insertar, entonces hubo esa chance de septiembre de 2014 hasta diciembre en la secretaría como becario y después me volvieron a llamar. $Y$ bueno un poco que te abren las puertas y después tenés que hacer lo tuyo (Tomás, de Tandil, comunicación personal).
\end{abstract}


Gonzalo Ricardo Mamani Caer en la universidad pública. Trayectorias educativas en el marco de la política pública del Albergue Transitorio

Por otra parte, Candela y Carolina decidieron volverse a sus respectivas provincias. La primera por una cuestión de arraigo a su pueblo y familia. Sin embargo pensó en quedarse en La Plata pero la escasa salida laboral y los altos costos para mantener la matrícula provincial del Colegio de Odontólogos, imposibilitaron y debilitaron sus ganas de permanecer en la ciudad. En tanto la segunda, trató de hacer todo para quedarse en La Plata, pero no encontró la forma puesto que considera que dedicarse al periodismo deportivo es difícil, a pesar de haber tenido varias experiencias, por lo que no tuvo alternativa de regresar a Chubut y actualmente continúa estudiando la carrera de Licenciatura en Letras.

\begin{abstract}
Me quede acá en La Plata este año porque mi idea era trabajar acá. No sé si siempre fue tan complicado. Uno sabe que el mundo del periodismo se mueve todo por contacto. La verdad que es bastante más difícil que alguien te quiera pagar por lo que haces y si bien no espero hacerme millonaria, pero queres que no te exploten. Hoy por hoy hago radio con un grupo de colegas, estoy escribiendo en una página, donde también lo pagamos. Todo lo que hago lo pagó, en realidad la página no, porque la paga uno de mis compañeros. Pero si, pagamos. Creo que si hoy por hoy tengo que estar en un lugar donde no me paguen para cumplir órdenes, no sé si lo hago. O no dentro del periodismo, quizás hago eso en un quiosco, así que me vuelvo allá a mis pagos a ver si es un poco menos complicado (Carolina, de Trelew, comunicación personal).
\end{abstract}

Matías considera que ante este contexto de ajuste en la Ciencia y Técnica a nivel nacional, tuvo la suerte de haber entrado como becario CONICET. Al momento de enterarse que había obtenido la beca, le faltaba un solo final de la carrera, por lo que decidió abandonar el AU y darle su lugar a otro/a estudiante. Comenta que en su Facultad la salida laboral -en términos globales- es la investigación y la docencia. En ese punto los recortes en el CONICET afectaron demasiado los cupos para ser becario.

Pía también tuvo una difícil salida del AU no por la salida laboral, sino lo que implicaba las horas de trabajo diario para poder cubrir los gastos mensuales. Actualmente comparte alquiler con una compañera para poder cubrir los gastos del mismo y en algunas oportunidades de suplencia tuvo que trabajar hasta diez horas por día. Los inicios de la docencia es algo complejo por la cantidad de puntaje docente y no tener antigüedad.

A raíz de estos relatos se abren nuevos interrogantes a los desafíos de esta política pública de inclusión universitaria, puesto que plantea una contención en varios frentes para la permanencia y egreso de los/as estudiantes. Sin embargo a la hora de la finalización de la carrera hay un enorme bache para la inserción en la ciudad y el mundo laboral. Son las carreras estratégicas las que tienen mayor salida laboral por su vacancia, en tanto las demás 
Gonzalo Ricardo Mamani Caer en la universidad pública. Trayectorias educativas en el marco de la política pública del Albergue Transitorio

las dificultades aumentan. Ante esta situación ¿habilita a nuevas transformaciones de la política pública del Albergue Universitario? ¿Cómo puede contribuir en la rápida inserción de los/as jóvenes en una coyuntura de ajuste económico?

\section{Consideraciones finales y continuación del proceso}

Se mencionó en este artículo, las trayectorias familiares de los/as jóvenes. En esa línea se pudo constatar la importancia en la significación para estos/as por ser, en su gran mayoría, la primera generación de estudiantes que accedieron a la universidad pública y obtuvieron un título de grado. Es de destacar que una década después de la peor crisis económica y política de nuestro país, surja una la política pública que posibilite a estudiantes con condiciones socio económica difíciles que puedan acceder a la universidad y en el caso de estos/as ocho chicos/as graduarse.

Por otro lado, se pudo conocer los múltiples procesos de comunicación/educación en la experiencia de los/as graduados/as en su tránsito por el AU. Asimismo se pueden sintetizar a partir de tres puntos fuertes: 1. reconocimiento de la política pública; 2. diálogo y debate de lo instituido 3. lucha por nuevos derechos. En cuanto al primero, una parte de los/as entrevistados/as vivieron una desde la inauguración de la política pública en 2011, en la cual experimentaron la etapa inicial cuando convivían 36 estudiantes. Reconocieron dónde y cómo vivían, ya que se generaron lazos de compañerismo y modos de hacer el $\mathrm{AU}$ en comunidad. Este momento inicial derivó al segundo punto, en el cual diversas transformaciones empezaron a darse, ya que a partir del diálogo y debate los/as estudiantes encararon el primero conflicto institucional en pos de mejorar las condiciones de convivencia impuestas por el organismo regulador de la beca. Por último, la ampliación de derechos donde se posibilitaron y ganaron por medio organización estudiantil clave para el bienestar de los/as becarios/as.

Estos procesos de comunicación/educación dan cuenta que el $\mathrm{AU}$, no es solo un lugar de convivencia diaria y una política pública de inclusión educativa, sino que es mucho más. Se producen condiciones para la producción y organización de nuevos conocimientos y aprendizajes en común y, que van más allá de los adquiridos en las distintas unidades académicas de la UNLP. Se trata de otros saberes que vienen desde las distintas trayectorias de quienes lo habitan y que se ponen en juego en los modos de apropiarse de este espacio. La heterogeneidad cultural que posee este lugar son demasiadas diversas y amplias, lo cual posibilita la continuidad de futuras indagaciones. En esta primera aproximación a lo que significa el $\mathrm{AU}$, se pudo reconocer que el años transitados por estos/as jóvenes, pudieron 
intervenirlo desde distintos lugares y dieron el puntapié inicial de lo que hoy es este derecho. Un edificio que estuvo muchos años abandonado, hoy se encuentra recargado de sentidos.

Para continuar con las experiencias de los/as graduados/as por su paso por el AU, es necesario detenerse en una cuestión clave: las nociones de la meritocracia. Hablar acerca de las políticas públicas de la cual fueron parte, algunos/as entrevistados/as hicieron hincapié desde una mirada meritocracia y selectiva de quienes pueden acceder y mantener este derecho o qué carreras tendrían que tener prioridad. Estas conceptualizaciones acerca de mérito los/as atraviesan transversalmente a partir de sus vivencias y experiencias donde se pudo dar cuenta internalización de estos conceptos. A pesar de valoración y reindivicación este tipo de derechos que fomenta la igualdad de oportunidad, la lógica meritocracia está presente. Otra cuestión fundamental que posibilita futuras nuevas investigaciones es la transformación que va adquiriendo esta política pública. Se desarrolló los diferentes cambios que fue teniendo el $\mathrm{AU}$ en cuanto a la convivencia y los derechos conquistados por los/as estudiantes. En esa línea, un nuevo emergente es sin dudas de la salida de la beca y la inserción laboral de los/as nuevos/as graduados/as. En el contexto actual es sumamente complejo independizarse desde lo económico habiendo obtención el título de grado recientemente. Se pudo indagar a partir de los relatos de los/as jóvenes que esto es una cuestión difícil y complicada de afrontar, en términos que este derecho brinda una contención en diversos aspectos. Sin embargo, cuando se trata de salir de la misma, consideran que vuelven a estar en las mismas condiciones en las que entraron, más allá de haber finalizado sus estudios universitarios. Ante esta situación algunos/as entrevistados/as sugirieron que quienes estén en frente de la gestión del AU puedan dar respuesta a esta situación. No obstante, no se encuentran en el misma escenario sino que están en las mismas condiciones que el resto de los/as graduados/as universitarios/as.

\section{Bibliografía}

AA/VV (2007) El sujeto de la educación. Sophía de la educación Colección de Filosofía de la educación N². Universidad Politécnica Salesiana Ecuador. Capítulo 2. Recuperado de https://sophia.ups.edu.ec/index.php/sophia/article/view/2.2007.05

AA/VV (2017) Cuaderno de Cátedra Comunicación y Educación. Cátedra 2. Facultad de Periodismo y Comunicación Social Universidad Nacional de La Plata. Recuperado de https://perio.un/p.edu.ar/catedras/system/files/compilacion cuaderno de catedra agosto 2017.pdf 
Gonzalo Ricardo Mamani Caer en la universidad pública. Trayectorias educativas en el marco de la política pública del Albergue Transitorio

Bourdieu, P. (1997b). La ilusión biográfica. En: Razones prácticas, sobre la teoría de la acción, Barcelona: Anagrama.

Buenfil Burgos, R. (1993) Análisis del Discurso y Educación. Departamento de Investigaciones Educativas Centro de Investigación y de Estudios Avanzados del Instituto Politécnico Nacional. México.

Bolis, J. (2014). Jóvenes y Soberanía Hegemonía, discursos y trayectorias hacia la emancipación. (Tesis de grado). Facultad de Periodismo y Comunicación Social, Universidad Nacional de La Plata.

Bourdieu, P. (1984). La 'juventud' no es más que una palabra. En Sociología y cultura. México: Grijalbo.

Camusso, L. (2015). Juventudes y ciudadanía: posibles aportes del Estado para el fortalecimiento del proceso de ciudadanización. Revista Argentina de Estudios de Juventud. Facultad de Periodismo y Comunicación Social, La Plata.

Carli, S. (2006). Los dilemas de la transmisión en el marco de la alteración de las diferencias intergeneracionales. Diploma Superior en Gestión Educativa de FLACSO. Recuperado de https://bit.ly/34oP970

Cifuentes Gil, R. (2011) Diseños de proyectos de investigación cualitativa. Centro de Publicaciones Educativas y Materiales Didácticos. Buenos Aires.

De Certeau, M. (1996). La invención de lo cotidiano I. Artes de hacer. México: Universidad Iberoamericana desplazado. Artículo presentado en VIII Congreso Latinoamericano de Investigadores de la Comunicación-ALAIC 2006, Universidade do Vale do Rio dos Sinos, São Leopoldo, Rio Grande do Sul, Brasil.

Díaz Larrañaga, N. (1999). El relato de una vida: apuntes teóricos metodológicos en comunicación. Revista Latina de Comunicación Social, 22.

Diker, G. (2008). Autoridad y transmisión: algunas notas teóricas para re-pensar la educación. Revista Educación y Humanismo, 15, Universidad Simón Bolívar, Barranquilla, Colombia, pp. 58-69.

Dubet, F. (2011). La experiencia sociológica. Barcelona: Gedisa.

Escobar, A. (2016). Sentipensar con la Tierra: Las Luchas Territoriales y la dimensión ontológica de las epistemologías del sur. AIBR revista de antropología iberoamericana, pp. 11-32. doi: 10.11156/aibr.110102

Ferrante, N. (2013). Jóvenes y tecnologías: relatos de experiencias a partir del Programa Conectar Igualdad. Question, 1(39), 4-10. Recuperado de https://bit.ly/38EhD0Q

Geertz, C. (2003). La interpretación de las culturas. Barcelona: Gedisa. 
Gonzalo Ricardo Mamani Caer en la universidad pública. Trayectorias educativas en el marco de la política pública del Albergue Transitorio

Giroux, H. (1990). Los profesores como intelectuales transformativos. En Los profesores como intelectuales. Hacia una pedagogía crítica del aprendizaje (capítulo 9). Buenos Aires: Paidós.

Glaser, B. y Strauss, A. (1967). The Discovery of Grounded Theory. Strategies for Qualitative Research. Chicago: Aldine.

Guber, R. (2009). El Salvaje Metropolitano: reconstrucción del conocimiento social en el trabajo de campo. Buenos Aires: Editorial Paidós.

Huergo, J. (comp.). (2009). Algunos desafíos a la comunicación/educación comunitaria y popular. En Construyendo comunidades... Reflexiones actuales sobre comunicación comunitaria, Área de Comunicación Comunitaria. Buenos Aires: La Crujía.

Huergo, J. (1997). Comunicación/Educación. Ámbitos, prácticas y perspectivas. La Plata: Ediciones de Periodismo y Comunicación Social, UNLP.

Kessler, G. (2002). La experiencia escolar fragmentada. Estudiantes y docentes en la escuela media de Buenos Aires. Buenos Aires: IIPE-UNESCO.

Kornblit, A. L. (2007). Metodologías cualitativas en ciencias sociales. Buenos Aires: Editorial Biblos.

Margulis, M. (2008). La juventud es más que una palabra. Ensayos sobre Cultura y Juventud. Editorial Biblos Sociedad. Buenos Aires.

Marradi, A.; Archenti, N. y Piovani, J. I. (2007). Metodología de las Ciencias Sociales. Buenos Aires: Emecé Editores.

Martin-Barbero, J. (2003). Saberes hoy: diseminaciones, competencias y transversalidades. Revista Iberoamericana de Educación, 32, pp. 17-34. Edición de la Organización de Estados Iberoamericanos para la Educación, la Ciencia y la Cultura (OEI), Centro de Altos Estudios Universitarios (CAEU), Madrid, España.

Martin-Barbero, J. (2009). Culturas y comunicación globalizada. I/C-Revista Científica de Información y Comunicación, 6, Secretariado de Publicaciones de la Universidad de Sevilla, pp-175-192.

Martínez, D. (2011). La educación primaria de jóvenes y adultos en La Plata. Un análisis de las subjetividades y las prácticas desde comunicación/educación. (Tesis de Maestría PLANGESCO). FPyCS, La Plata. Recuperado de http://sedici.unlp.edu.ar/handle/10915/22447 
Gonzalo Ricardo Mamani Caer en la universidad pública. Trayectorias educativas en el marco de la política pública del Albergue Transitorio

Martínez, D. (2015). Saberes, experiencias y subjetividades de la educación de jóvenes y adultos en la ciudad de La Plata. Un abordaje desde la perspectiva de comunicación/educación. (Tesis Doctoral). FPyCS, La Plata. Recuperado de http://sedici.un|p.edu.ar/handle/10915/49538

Mazzola, R. (2014). PROGRESAR: Juventudes, bienes públicos y justicia distributiva. Revista Estado y Políticas Públicas, 2. Facultad Latinoamericana de Ciencias Sociales (FLACSO).

Mc Laren, P. (1995) La escuela como performance ritual. Hacia una economía política de los símbolos y gestos educativos, México, Siglo XXI

Núñez, P. (2013). La política en la escuela: jóvenes, justicia y derechos en el espacio escolar. Buenos Aires: La Crujía.

Paiva, Juan Bautista (2016) Los sentidos sobre la educación de los jóvenes destinatarios del PROGRESAR en La Plata. TIF de grado. Facultad de Periodismo y Comunicación Social UNLP

Southwell, M. (2009). Docencia, tradiciones y nuevos desafíos. En Yuni, J. (comp.). La formación Docente. Complejidad y ausencias. Catamarca: Grupo Editor-Facultad de Humanidades, Universidad Nacional de Catamarca.

Reguillo, R. (2013). Culturas Juveniles: formas políticas del desencanto. Buenos Aires: Siglo Veintiuno Editoriales.

Ros, M.; Benito, L.; Germain, L. y Justianovich, S. M. (2017). Inclusión, trayectorias estudiantiles y políticas académicas en la universidad. La Plata: EDULP. Recuperado de http://sedici.unlp.edu.ar/handle/10915/64308

Saintout, F. (2003). Abrir la comunicación. Tradición y movimiento del campo académico. La Plata: Ediciones de Periodismo y Comunicación.

Saintout, F. (2007). Los estudios socioculturales y la comunicación. Un mapa Un mapa desplazado. Artículo presentado en VIII Congreso Latinoamericano de Investigadores de la Comunicación - ALAIC 2006, Universidade do Vale do Rio dos Sinos, São Leopoldo, Rio Grande do Sul, Brasil.

Saintout, F. (directora) (2015). Conectar Igualdad. Apropiaciones de las netbooks por fuera del ámbito escolar. Centro de Investigaciones en problemáticas socio simbólicas latinoamericanas Aníbal Ford. Dirección de Publicaciones Científicas: La Plata, Argentina. Recuperado de https://bit.ly/38JgLlf

Saintout, F. (coordinadora) (2016). Juventudes argentinas: prácticas culturales, ciudadanía y participación. Ciudad Autónoma de Buenos Aires: Grupo Editor Universitario. 
Gonzalo Ricardo Mamani Caer en la universidad pública. Trayectorias educativas en el marco de la política pública del Albergue Transitorio

Souza, M. S.; Giordano, C. y Migliorati, M. (2012). Hacia la Tesis. Itinerarios conceptuales y metodológicos para la investigación en comunicación. Instituto de Investigación en Comunicación IICOM, FPyCS, UNLP, La Plata. Recuperado de http://sedici.unlp.edu.ar/handle/10915/42082

Sousa Santos (s/f). Introducción: Las Epistemologías del Sur. Recuperado de https://bit.ly/34qRejl

Terigi, F. (2008). Los cambios en el formato de la escuela secundaria argentina: por qué son necesarios, por qué son tan difíciles. Propuesta Educativa, 15(29), Vol 1.

Thompson, E. P. (1963). La conciencia de clase. En La formación de la clase obrera en Inglaterra (pp. 313-452). Barcelona: Crítica.

Tobeña, V. (2011). La escuela en el mundo contemporáneo. Notas sobre el cambio cultural. En Variaciones sobre la forma escolar. Límites y posibilidades de la escuela media. Buenos Aires: Homosapiens.

Urresti, M. (2015). Nómades, convergentes, protésicos y obnubilados. Los jóvenes ante la emergencia del campo tecnológico digital. En Quevedo, L. A. (Comp.). La cultura argentina hoy: Tendencias. Buenos Aires: Siglo XXI Editores. 